\title{
Strain Analysis of the de Mattia Test
}

\author{
Ch. Feichter ${ }^{1}$, Sz. Vezer ${ }^{1}$, M. Reiter ${ }^{2}$ and Z. Major ${ }^{2}$ \\ ${ }^{1}$ Polymer Competence Center Leoben GmbH, A-8700 Leoben Rosegger Str 12, Austria \\ ${ }^{2}$ Institute of Polymer Product Engineering, Johannes Kepler University Linz, Austria
}

\begin{abstract}
The de Mattia test is a well-known, standardized and widely used method in the rubber industry for characterizing the fatigue behaviour of rubbers. Due to the visual observation and classification of the crack initiated, high data scatter were usually observed in these tests. To improve the quality of the de Mattia test and to support the applicability of the test method in modern design procedures, two novel experimental methods were proposed. Full-field strain analysis experiments using digital image correlation technique were performed and the local strains at the notch tip determined in the first. A global displacement vs. local strain calibration curves makes the design and conduction of strain based Wöhler curves possible. The crack initiation and crack growth is detected by an image analysis system and the crack growth rate was determined in the second method. To gain more insight into the fatigue behaviour of rubbers, these two novel methods were combined and can efficiently be used for characterizing the fatigue behaviour of rubbers.
\end{abstract}

\section{Introduction and Objectives}

In engineering applications elastomers are frequently exposed to complex combinations of mechanical loads (monotonic, static, intermittent and cyclic loads). A better understanding of the material resistance against crack initiation and propagation becomes of increasing practical importance. Various methods were developed and used for characterizing the fatigue behaviour of elastomers. The de Mattia test is a well-known and widely used method in the rubber industry for characterizing the fatigue behaviour of rubbers [1]. The method is standardized in various standards (ISO 132, ASTMD 430 and D813). The specimen is a rubber strip with a smooth notch $(\mathrm{R}=5 \mathrm{~mm})$ and loaded by the linear movement of the testing machine up to $57 \mathrm{~mm}$ stroke level. This movement results in large strain global bending deformations of the rubber strip and also causes high strain concentration at the notch tip. It is assumed that this type of deformation is similar to which occur in many elastomeric parts (e.g., tire side wall, air spring and belts) during the service loading. Due to the displacement controlled loading, simultaneously more specimens (in general 6 specimens in a linear set-up) can be investigated and hence, the statistical nature of the rubber fatigue can also be adequately described. However, to improve the applicability of this test method, the basic procedure should be further developed. The definition, determination and classification of the crack length are based on a subjective judgement which makes the comparability of various results unreliable and difficult. Furthermore, no accurate information about the local strain state is available and the comparison of the results with the results of other fatigue tests is hardly possible. Hence, the objectives of this study were; (a) to determine the local

This is an Open Access article distributed under the terms of the Creative Commons Attribution-Noncommercial License 3.0, which permits unrestricted use, distribution, and reproduction in any noncommercial medium, provided the original work is properly cited. 
strain distribution of the de Mattia specimen during the standard loading process and (b) to characterize the crack initiation in the de Mattia specimen on an objective basis.

\section{Experimental}

Three different tests were performed on 2 rubber grades in this study using the de Mattia specimen and test set-up:

- Monotonic tests up to $57 \mathrm{~mm}$ global displacement and with simultaneous full-field strain analysis.

- Conventional single and multiple specimen de Mattia tests under cyclic loading conditions

- $\quad$ Single specimen fatigue tests with image data acquisition.

The rubbers (rubber 1 and 2) are produced and provided in form of de Mattia test specimen for these experiments by the Semperit Technische Produkte Ges.m.b.H (Wimpassing, A). Single specimen experiments were performed on a servohydraulic (MTS 858, MTS System Berlin, D) testing machine and multiple specimen tests on a de Mattia test system (Semperit, Wimpassing, A). The full-field strain was measured using a digital image correlation test system (Aramis, GOM, Braunschweig, D) and 3D strain analysis was carried out. This technique was previously developed, successfully applied for other rubber specimens and described in [1]. The full-field strain analysis image of the front view of loaded specimen ( $30 \mathrm{~mm}$ global deformation) is shown in figure 1. Sections were defined in this image at the notch tip in transverse direction and in vertical direction. The Major strain values along with these sections for increasing global displacement values are plot in figures 2 and 3 for section 1 and section 2 respectively. With increasing global displacement up to $57 \mathrm{~mm}$ increasing local strain values were observed in both sections. The maximal major strain values are in the range of 100 to $110 \%$. Full-field strain analysis was also performed on the side view of the specimen with similar procedure and the corresponding image is shown in figure 4. Due to the large deformation and the relatively low thickness (smaller range of interest, RoI) of the specimen the quality of these images are lower than for the front view images. In spite of this fact, similar local strain values were determined and are shown in figure 5.

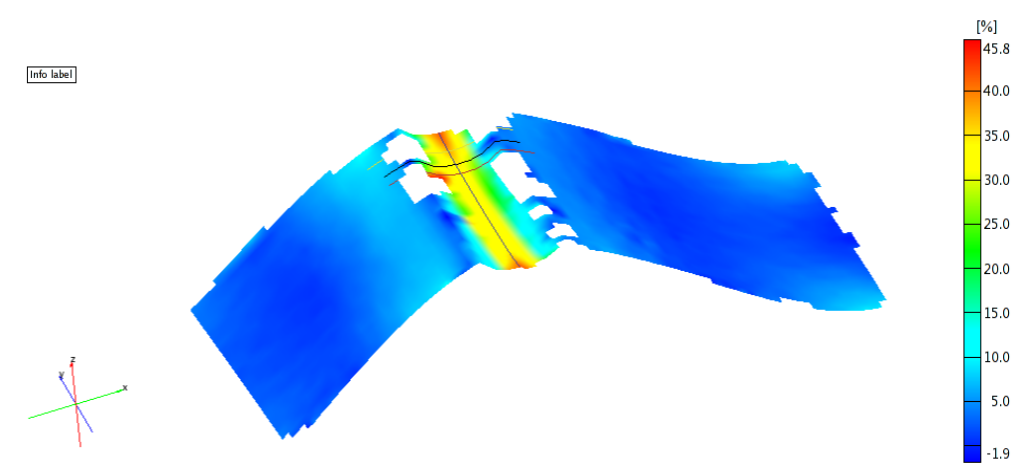

Fig. 1. Full-field strain analysis of the de Mattia specimen. Front view. 
14th International Conference on Experimental Mechanics

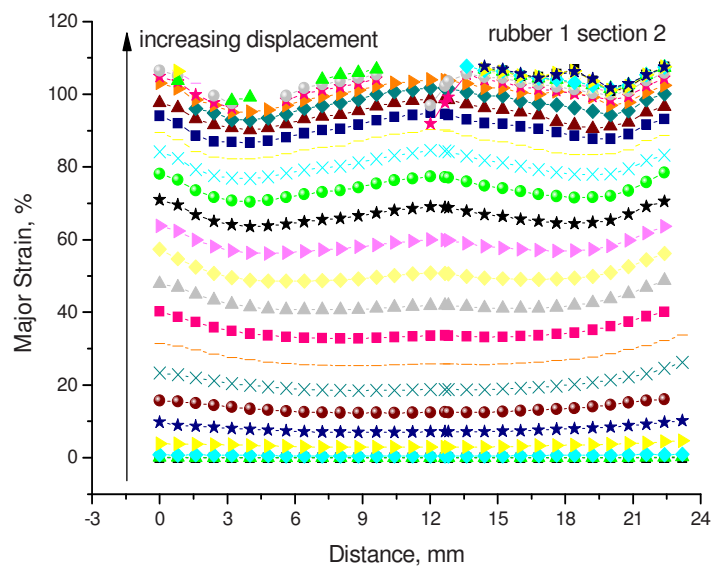

Fig. 2. Global displacement dependence of the Major Strain in the de Mattia specimen.

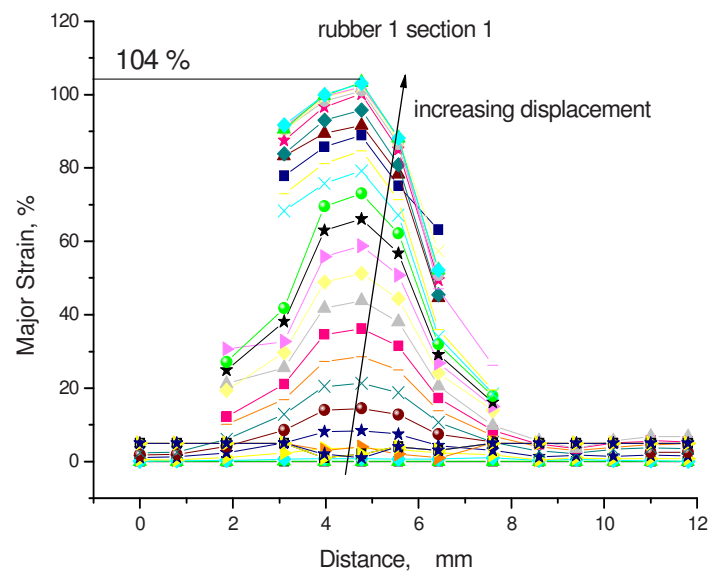

Fig. 3. Major strain values along with section 1 in the notch.

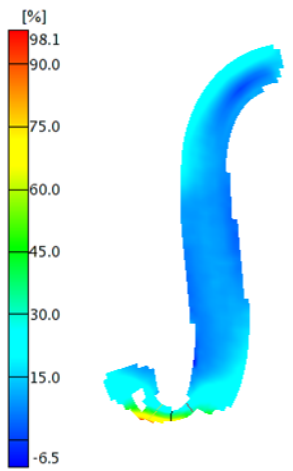

Fig. 4. Full-field strain analysis of the de Mattia specimen. Side view. 


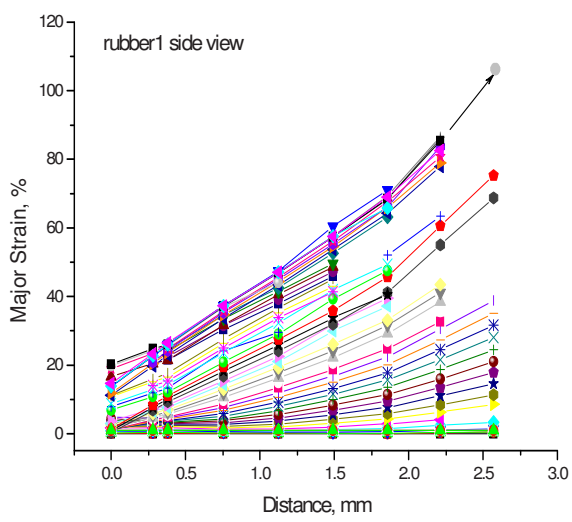

Fig. 5. Global displacement dependence of the Major Strain in the de Mattia specimen. Side view.

Furthermore, the conventional cyclic tests were carried out with visual observation and classification of the crack according to the relevant standards. To get more insight into the crack initiation and crack growth process a digital image acquisition system was also applied. A special trigger procedure was developed and implemented in the software. This procedure makes the determination of force-global displacement and local displacement values without phase shift. The procedure along with some examples is described in more detail in [4].

\section{Results and Discussion}

Cyclic de Mattia tests were performed using single specimens (testing machine 1) and multiple specimen (testing machine 2). The tests were stopped and the crack was identified and classified by visual observation. The results of these tests are shown in figure 6. A relatively high data scatter, in the range of a half decade was observed. To improve the quality and applicability of the de Mattia tests two novel methods are proposed. While method 1 uses the results of the full-field strain measurements, the crack growth kinetics is determined by an image data acquisition system in method 2.

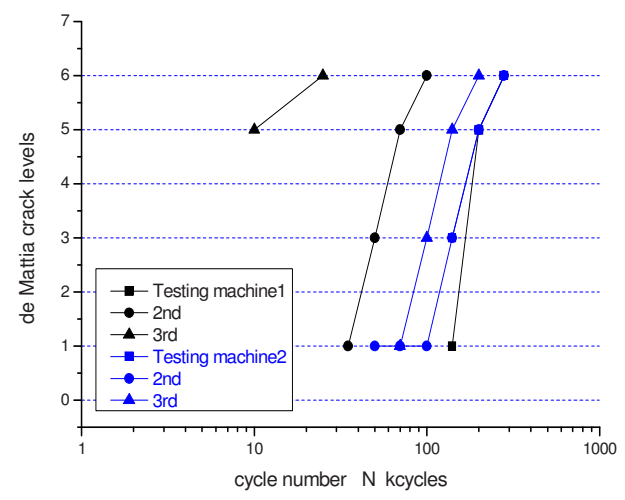

Fig. 6. Cycle number dependence of the crack levels identified during the cyclic tests for the de Mattia specimen. Visual observation. 
Based on the local strain measurements a global displacement vs. local strain calibration curve was established for both rubber types and these curves are shown in figure 7. A non-linear relationship between global displacement and local strain was observed for both rubber grades. Based on these calibration curves local strain based Wöhler curves could be constructed. This procedure is described in more detail elsewhere [3].

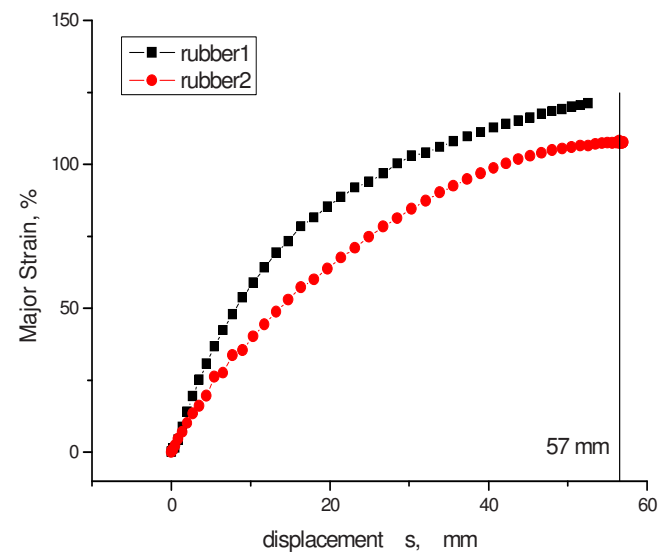

Fig. 7. Global displacement vs. local Major Strain curves for the de Mattia specimen.
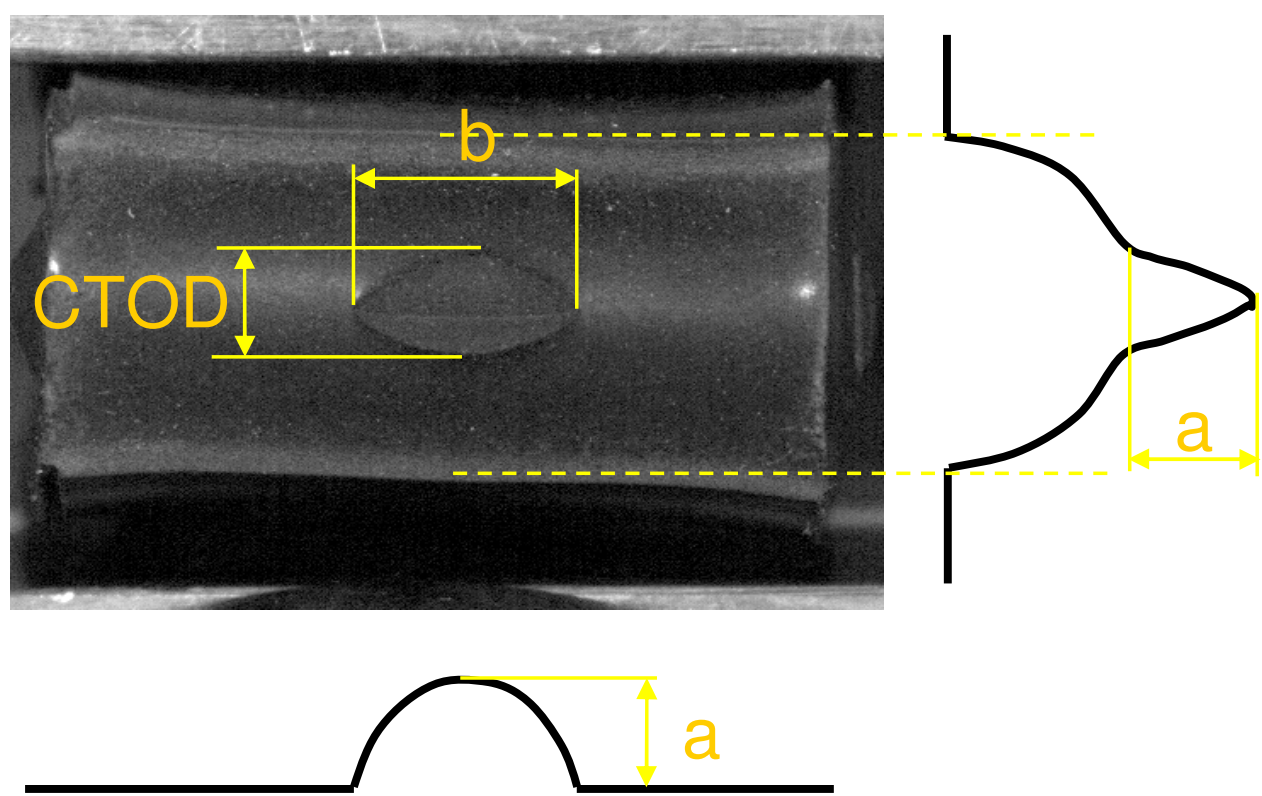

Fig. 8. Schematic illustration of the crack length measurement for de Mattia specimen. Image data acquisition. 


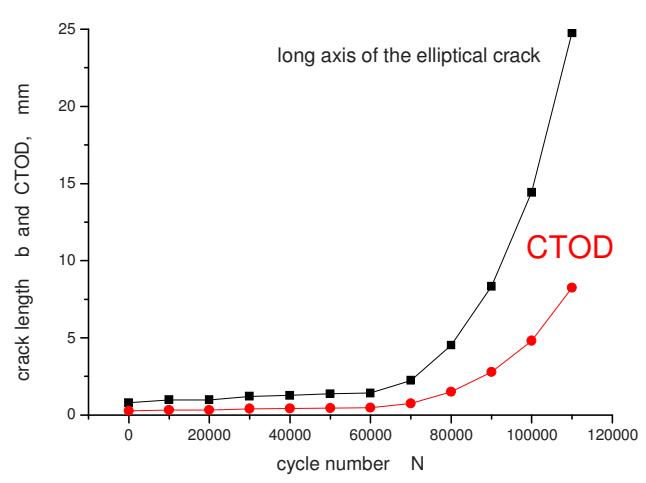

Fig. 9. Crack growth curves (b and CTOD) for the de Mattia specimen.

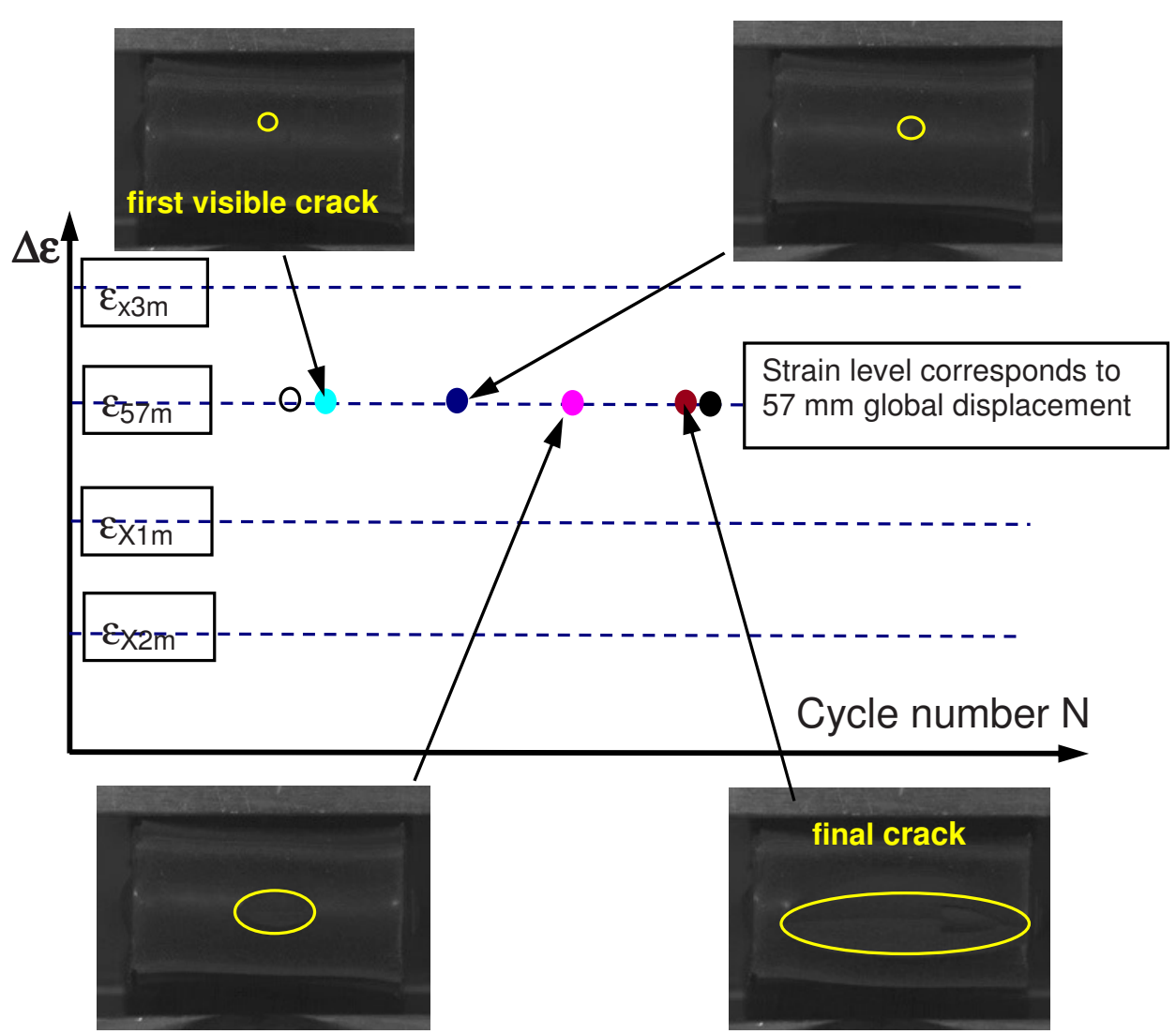

Fig. 10. Schematic representation of the combination of crack growth rate measurement and local strain controlled Wöhler curves. 
The crack can be detected by the image data acquisition system. An elliptical crack was observed at the notch tip of the specimen. The schematic representation of the crack length determination procedure is shown in figure 8 . The major axis of the ellipsis is called as $b$, the minor axis as the crack tip opening displacement and the crack length, a, is associated with the crack in thickness direction of the specimen. The cycle number dependence of the crack growth in terms of $b$ and CTOD is shown in figure 9. Similar crack growth curves were observed as for other fracture mechanics experiments (i.e., pure shear specimens). Based on these curves the crack growth rate, $\mathrm{da} / \mathrm{dN}$ can also be calculated for further analysis.

The local strain based Wöhler curve concept and the crack growth kinetics measurement can be combined. The schematic representation of this combination is shown in figure 10. This combination makes the link between the conventional Wöhler type fatigue analysis methods and fracture mechanics methods possible.

\section{Conclusions and Outlook}

Three different tests were performed on 2 rubber grades in this study using the de Mattia specimen and test set-up:

- Monotonic tests up to $57 \mathrm{~mm}$ global displacement and with simultaneous full-field strain analysis were carried out. The local strain at the notch tip were determined and a calibration curves between global displacement and local strain was established

- Conventional standardized single and multiple specimens de Mattia tests under cyclic loading conditions were performed and evaluated.

- $\quad$ Single specimen fatigue tests with image data acquisition were performed and the crack initiation and the crack growth were measured.

The global displacement-local strain calibration curve makes the construction of strain based Wöhler curve possible. This method is useful for characterizing the fatigue behaviour of elastomers and can be linked with numerical methods for component design. Finally, the conventional de Mattia test procedure can effectively be completed with the combination of the determination of local strain based Wöhler curves and crack growth measurements.

\section{References}

1. ASTM D 430-95 and ASTM D813-95, Standard test method for rubber deterioration. Fatigue and crack growth.

2. Feichter, Ch., Major, Z. and Lang, R.W., Strain, 42, pp. 299-304 (2006)

3. Major, Z., Construction of local strain based Wöhler curves for characterizing the fatigue behavior of elastomers, in preparation.

4. Vezer, Sz. and Major, Z., Materials Engineering (UZ), Analogue triggered image acquisition method for optical measurement under cyclic load conditions, 1/2008, 8, pp. 0513.

\section{Acknowledgments}

This project was performed at the Polymer Competence Center Leoben $\mathrm{GmbH}$ within the Kplus programme of the Austrian Ministry of Traffic, Innovation and Technology. The funding within this programme by the Governments of Austria, Styria and Upper Austria is gratefully acknowledged. Special appreciation goes to the SEMPERIT Technische Produkte Gesellschaft.m.b.H (Wimpassing, A) for producing the specimens, performing tests and for the valuable discussion with Mr. W. Fidi, Dr. A. Holzner, Mr. S. Pollaschek and Ms. S. Koch. 